\title{
Article
}

\section{On some properties of generalized Fibonacci polynomials}

\section{Fidel Oduol}

Department of Pure and Applied Mathematics, Maseno University, Private Bag, 40105, Maseno-Kenya.; ochieng.fidel@yahoo.com

Received: 23 July 2020; Accepted: 1 September 2020; Published: 23 September 2020.

\begin{abstract}
Fibonacci polynomials have been generalized mainly by two ways: by maintaining the recurrence relation and varying the initial conditions and by varying the recurrence relation and maintaining the initial conditions. In this paper, both the recurrence relation and initial conditions of generalized Fibonacci polynomials are varied and defined by recurrence relation as $R_{n}(x)=a x R_{n-1}(x)+b R_{n-2}(x)$ for all $n \geq 2$, with initial conditions $R_{0}(x)=2 p$ and $R_{1}(x)=p x+q$ where $a$ and $b$ are positive integers and $p$ and $q$ are non-negative integers. Further some fundamental properties of these generalized polynomials such as explicit sum formula, sum of first $n$ terms, sum of first $n$ terms with (odd or even) indices and generalized identity are derived by Binet's formula and generating function only.
\end{abstract}

Keywords: Generalized Fibonacci polynomials, Binet's formula, generating function.

MSC: 11B37, 11B39.

\section{Introduction}

$\mathbf{F}$ ibonacci polynomials [1] are special cases of Chebyshev polynomials and are defined recursively by

$$
F_{n}(x)=x F_{n-1}(x)+F_{n-2}(x),
$$

for all $n \geq 2$ with $F_{0}(x)=0$ and $F_{1}(x)=1$.

Lucas polynomials [1] are defined by

$$
L_{n}(x)=x L_{n-1}(x)+L_{n-2}(x),
$$

for all $n \geq 2$ with $L_{0}(x)=2$ and $L_{1}(x)=x$.

Pell polynomials [2] are defined by

$$
P_{n} x=2 x P_{n-1}(x)+P_{n-2}(x),
$$

for all $n \geq 2$ with $P_{0}(x)=0$ and $P_{1}(x)=1$.

Generating function of Fibonacci polynomials is given by

$$
\sum_{n=0}^{\infty} F_{n}(x) t^{n}=\frac{t}{1-x t-t^{2}}
$$

Explicit sum formula for Fibonacci polynomials is given by

$$
F_{n}(x)=\stackrel{\left\lfloor\frac{n-1}{2}\right\rfloor}{\sum_{k=0}^{n-k-1}}\left(\begin{array}{c}
n-1 \\
k
\end{array}\right) x^{n-2 k-1} .
$$

Horadam polynomials sequence [3] $h_{n}(x)$, for $n \geq 3$ is defined by recurrence relations $h_{n}(x)=$ $p x h_{n-1}(x)+q h_{n-2}(x)$, with initial conditions $h_{1}(x)=a$ and $h_{2}(x)=b x$, where $p, q, a$ and $b$ are integers. 
Generalized Fibonacci polynomials and some identities [4] are defined by

$$
w_{n}(x)=x w_{n-1}(x)+w_{n-2}(x),
$$

for $n \geq 2$ with $w_{0}(x)=2 b$ and $w_{1}(x)=a+b$, where $a$ and $b$ are integers.

Generalized Fibonacci-Like polynomials [5] are defined by the recurrence relation:

$$
m_{n}(x)=x m_{n-1}(x)+m_{n-2}(x),
$$

with $m_{0}(x)=2 s$ and $m_{1}(x)=1+s$, where $s$ is an integer.

Fibonacci-like polynomials [6] are defined by the recurrence relation

$$
S_{n}(x)=x S_{n-1}(x)+S_{n-2}(x),
$$

$n \geq 2$ with $S_{0}(x)=2$ and $S_{1}(x)=2 x$.

Generalized Fibonacci polynomials [7] are defined by the recurrence relation

$$
b_{n}(x)=x b_{n-1}(x)+b_{n-2}(x),
$$

$n \geq 2$ with $b_{0}(x)=2 b$ and $b_{1}(x)=s$, where $b$ and $s$ are integer.

In this paper, generalized Fibonacci polynomials is studied by varying both the recurrence relation and initial conditions. The properties of these polynomials are derived by means of Binet's formula and generating function. Few terms of generalized Fibonacci polynomials and characteristic equation of the recurrence relation are presented in Section 2. In Section 3 Binet's formula is obtained and generating function is also obtained in Section 4. Further some properties of these polynomials are presented in Section 5 and finally in Section 6 conclusion is given.

\section{Generalized Fibonacci Polynomials}

We define generalized Fibonacci polynomials by the recurrence relation

$$
R_{n}(x)=a x R_{n-1}(x)+b R_{n-2}(x), n \geq 2,
$$

with initial conditions $R_{0}(x)=2 p$ and $R_{1}(x)=p x+q$ where $a$ and $b$ are positive integers and $p$ and $q$ are non negative integers.

Few terms of generalized Fibonacci sequence are as follows:

$$
\begin{aligned}
& R_{0}(x)=2 p \\
& R_{1}(x)=p x+q \\
& R_{2}(x)=a p x^{2}+a q x+2 b p \\
& R_{3}(x)=a^{2} p x^{3}+a^{2} q x^{2}+2 a b p x+b p x+b q \\
& R_{4}(x)=a^{3} p x^{4}+a^{3} q x^{3}+2 a^{2} b p x^{2}+a b p x^{2}+a b q x+a b p x^{2}+a b q x+2 b^{2} p
\end{aligned}
$$

and so on.

The characteristic equation for the recurrence relation (6) is

$$
t^{2}-a x t-b=0
$$

This equation has two real and distinct roots;

$$
\alpha(x)=\frac{a x+\sqrt{a^{2} x^{2}+4 b}}{2}
$$

and

$$
\beta(x)=\frac{a x-\sqrt{a^{2} x^{2}+4 b}}{2} .
$$


Also from these roots, we have

$$
\begin{aligned}
\alpha(x) \beta(x) & =-b, \\
\alpha(x)+\beta(x) & =a x \\
\alpha(x)-\beta(x) & =\sqrt{a^{2} x^{2}+4 b} \\
\alpha^{2}(x)+\beta^{2}(x) & =a^{2} x^{2}+2 b, \\
\alpha^{2}(x) & =a x \alpha(x)+b, \\
\beta^{2}(x) & =a x \beta(x)+b .
\end{aligned}
$$

Generalized Fibonacci polynomials (6) generalizes, Fibonacci polynomials, Lucas polynomials and Pell polynomials at different values of $a, b, p$ and $q$.

- For $a=b=q=1$ and $p=0$, we obtain Fibonacci polynomials.

- For $a=b=p=1$ and $q=0$, we obtain Lucas polynomials.

- For $a=2, b=q=1$ and $p=0$, we obtain Pell polynomials.

Further for $x=1$ we obtain the corresponding sequences of these polynomials.

\section{Binet's formula for generalized Fibonacci polynomials.}

Theorem 1 (Binet's formula). The $n^{\text {th }}$ term of generalized Fibonacci polynomials is given by

$$
R_{n}(x)=A \alpha^{n}(x)+B \beta^{n}(x)=\frac{1}{\alpha(x)-\beta(x)}\left[R_{1}(x)\left(\alpha^{n}(x)-\beta^{n}(x)\right)+b R_{0}(x)\left(\alpha^{n-1}(x)-\beta^{n-1}(x)\right)\right]
$$

Proof. The characteristics Equation (7) has real and distinct roots. The solution of the recurrence relation (6) is therefore of the form

$$
R_{n}(x)=A \alpha^{n}(x)+B \beta^{n}(x),
$$

where $A$ and $B$ are constants and $\alpha(x)=\frac{a x+\sqrt{a^{2} x^{2}+4 b}}{2}$ and $\beta(x)=\frac{a x-\sqrt{a^{2} x^{2}+4 b}}{2}$.

Setting $n=0$ and $n=1$ in (9), we obtain

$$
A+B=R_{0}(x)
$$

and

$$
A \alpha(x)+B \beta(x)=R_{1}(x)
$$

respectively.

Solving these equations simultaneously, we obtain

$$
A=\frac{R_{1}(x)-\beta(x) R_{0}(x)}{\alpha(x)-\beta(x)}
$$

and

$$
B=\frac{\alpha(x) R_{0}(x)-R_{1}(x)}{\alpha(x)-\beta(x)}
$$

Substituting for $A$ and $B$ in (9), we get

$$
\begin{aligned}
R_{n}(x) & =\left(\frac{R_{1}(x)-\beta(x) R_{0}(x)}{\alpha(x)-\beta(x)}\right) \alpha^{n}(x)-\left(\frac{R_{1}(x)-\alpha(x) R_{0}(x)}{\alpha(x)-\beta(x)}\right) \beta^{n}(x) \\
& =\frac{1}{\alpha(x)-\beta(x)}\left[R_{1}(x)\left(\alpha^{n}(x)-\beta^{n}(x)\right)+b R_{0}(x)\left(\alpha^{n-1}(x)-\beta^{n-1}(x)\right)\right] .
\end{aligned}
$$

Hence the proof.

Remark 1. We have that

$$
A+B=R_{0}(x),
$$




$$
\begin{gathered}
A \alpha(x)+B \beta(x)=R_{1}(x), \\
A \beta(x)+B \alpha(x)=a x R_{0}(x)-R_{1}(x), \\
A \beta^{2}(x)+B \alpha^{2}(x)=a^{2} x^{2} R_{0}(x)-a x R_{1}(x)+b R_{0}(x),
\end{gathered}
$$

and

$$
A B=\frac{R_{0}(x) R_{2}(x)-R_{1}^{2}(x)}{\left(\alpha(x)^{-} \beta(x)\right)^{2}} .
$$

\section{Generating function for generalized Fibonacci polynomials}

Theorem 2 (Generating function). Generating function for generalized Fibonacci polynomials is given by

$$
\sum_{n=0}^{\infty} R_{n}(x) t^{n}=\frac{2 p+t[(p x+q)-2 a x p]}{1-a x t-b t^{2}} .
$$

Proof. Applying power series to the generalized Fibonacci polynomial $\sum_{n=0}^{\infty} R_{n}(x) t^{n}$, we have

$$
2 p+(p x+q) t+\left(a x^{2} p+a x q+2 b p\right) t^{2}+\cdots=\sum_{n=0}^{\infty} R_{n}(x) t^{n} .
$$

Now, multiplying the generating series by $\left(1-a x t-b t^{2}\right)$, where $\left(1-a x t-b t^{2}\right) \neq 0$, we get

$$
\begin{aligned}
& \left(1-a x t-b t^{2}\right) \sum_{n=0}^{\infty} R_{n}(x) t^{n}=\sum_{n=0}^{\infty} R_{n}(x) t^{n}-a x \sum_{n=0}^{\infty} R_{n}(x) t^{n+1}-b \sum_{n=0}^{\infty} R_{n}(x) t^{n+2} \\
& =\left[R_{0}(x)+R_{1}(x) t+\sum_{n=2}^{\infty} R_{n}(x) t^{n}\right]-a x\left[R_{0}(x) t+\sum_{n=2}^{\infty} R_{n-1}(x) t^{n}\right]-b \sum_{n=2}^{\infty} R_{n-2}(x) t^{n} \\
& =R_{0}(x)+\left[R_{1}(x)-a x R_{0}(x)\right] t+\sum_{n=2}^{\infty}\left[R_{n}(x)-a x R_{n-1}(x)-b R_{n-2}(x)\right] t^{n} \\
& =2 p+[(p x+q)-2 a p x] t+\sum_{n=2}^{\infty}\left[a x R_{n-1}(x)+b R_{n-2}(x)-a x R_{n-1}(x)-b R_{n-2}(x)\right] \\
& =2 p+[(p x+q)-2 a p x] t .
\end{aligned}
$$

Therefore

$$
\left(1-a x t-b t^{2}\right) \sum_{n=0}^{\infty} R_{n}(x) t^{n}=2 p+[(p x+q)-2 a p x] t
$$

Hence

$$
\sum_{n=0}^{\infty} R_{n}(x) t^{n}=\frac{2 p+[(p x+q)-2 a p x] t}{1-a x t-b t^{2}} .
$$

Remark 2. If $a=b=q=1$ and $p=0$ in (15), we obtain generating functions for Fibonacci polynomials (4).

\section{Some properties of generalized Fibonacci polynomials}

In this section, we obtain some properties of generalized polynomials by means of Binet's formula and generating function.

Proposition 1 (Explicit sum formula). Let $R_{n}(x)$ be the $n^{\text {th }}$ generalized Fibonacci polynomials, then 


$$
R_{n}(x)=2 p \sum_{k=0}^{\left\lfloor\frac{n}{2}\right\rfloor}\left(\begin{array}{c}
n-k \\
k
\end{array}\right)+(a x)^{n-2 k} b^{k}+(p x+q-2 a p x) \sum_{k=0}^{\left\lfloor\frac{n-1}{2}\right\rfloor}\left(\begin{array}{c}
n-k-1 \\
k
\end{array}\right)(a x)^{n-2 k-1} b^{k}
$$

where $\lfloor n\rfloor$ is the greatest integer less than or equal to $n$.

Proof. By generating function (15), we have

$$
\begin{aligned}
\sum_{n=0}^{\infty} R_{n}(x) t^{n} & =\frac{2 p+(p x+q-2 a p x) t}{1-a x t-b t^{2}} \\
& =[2 p+(p x+q-2 a p x) t][1-(a x+b t) t]^{-1} \\
& =[2 p+(p x+q-2 a p x) t] \sum_{n=0}^{\infty}(a x+b t)^{n} t^{n} \\
& =[2 p+(p x+q-2 a p x) t] \sum_{n=0}^{\infty} t^{n} \sum_{k=0}^{n}\left(\begin{array}{l}
n \\
k
\end{array}\right)(a x)^{n-k}(b t)^{k} \\
& =[2 p+(p x+q-2 a p x) t] \sum_{n=0}^{\infty} \sum_{k=0}^{n} \frac{n !}{k !(n-k) !}(a x)^{n-k} b^{k} t^{n+k}
\end{aligned}
$$

Now replacing $n$ with $n+k$, we get

$$
\begin{aligned}
\sum_{n=0}^{\infty} R_{n}(x) t^{n} & =[2 p+(p x+q-2 a p x) t] \sum_{n=0}^{\infty} \sum_{k=0}^{\infty} \frac{(n+k) !}{k ! n !}(a x)^{n} b^{k} t^{n+2 k} \\
& =[2 p+(p x+q-2 a p x) t] \sum_{n=0}^{\infty} \sum_{n=0}^{\left\lfloor\frac{n}{2}\right\rfloor} \frac{(n-k) !}{k !(n-2 k) !}(a x)^{n-2 k} b^{k} t^{n}
\end{aligned}
$$

Thus the sum equals to

$\sum_{n=0}^{\infty}\left[2 p \sum_{k=0}^{\left\lfloor\frac{n}{2}\right\rfloor} \frac{(n-k) !}{k !(n-2 k) !}(a x)^{n-2 k} b^{k}\right] t^{n}+\sum_{n=0}^{\infty}\left[(p x+q-2 a p x) \sum_{k=0}^{\left\lfloor\frac{n}{2}\right\rfloor} \frac{(n-k) !}{k !(n-2 k) !}(a x)^{n-2 k} b^{k}\right] t^{n+1}$.

Equating the coefficient of $t^{n}$ on both sides, we obtain

$$
R_{n}(x)=2 p \sum_{k=0}^{\left\lfloor\frac{n}{2}\right\rfloor}\left(\begin{array}{c}
n-k \\
k
\end{array}\right)(a x)^{n-2 k} b^{k}+(p x+q-2 a p x) \sum_{k=0}^{\left\lfloor\frac{n-1}{2}\right\rfloor}\left(\begin{array}{c}
n-k-1 \\
k
\end{array}\right)(a x)^{n-2 k-1} b^{k} .
$$

Proposition 2 (Sum of first $n$ terms). The sum of the first $n$ terms of generalized Fibonacci polynomials is given by

$$
\sum_{k=0}^{n-1} R_{k}(x)=\frac{R_{n}(x)+b R_{n-1}(x)-\left(R_{1}(x)-a x R_{0}(x)\right)-R_{0}(x)}{a x+b-1} .
$$

Proof. Using Binet's formula (8), we have

$$
\sum_{k=0}^{n-1} R_{k}(x)=\sum_{k=0}^{n-1}\left(A \alpha^{k}(x)+B \beta^{k}(x)\right)
$$

where $A=\frac{R_{1}(x)-\beta(x) R_{0}(x)}{\alpha(x)-\beta(x)}$ and $B=\frac{\alpha(x) R_{0}(x)-R_{1}(x)}{\alpha(x)-\beta(x)}$. It follows that 


$$
\begin{aligned}
\sum_{k=0}^{n-1} R_{k}(x) & =A \sum_{k=0}^{n-1} \alpha^{k}(x)+B \sum_{k=0}^{n-1} \beta^{k}(x) \\
& =\frac{A\left(\alpha^{n}(x)-1\right)}{\alpha(x)-1}+\frac{B\left(\beta^{n}(x)-1\right)}{\beta(x)-1} \\
& =\frac{A+B-(A \beta(x)+B \alpha(x))-\left(A \alpha^{n}(x)+B \beta^{n}(x)\right)}{\alpha(x) \beta(x)-\alpha(x)-\beta(x)+1}+\frac{\alpha(x) \beta(x)\left(A \alpha^{n-1}(x)+B \beta^{n-1}(x)\right)}{\alpha(x) \beta(x)-\alpha(x)-\beta(x)+1} .
\end{aligned}
$$

Since $\alpha(x)+\beta(x)=a x$ and $\alpha(x) \beta(x)=-b$ and using (8), (10) and (12), we obtain

$$
\sum_{k=0}^{n-1} R_{k}(x)=\frac{R_{n}(x)+b R_{n-1}(x)-\left(R_{1}(x)-a x R_{0}(x)\right)-R_{0}(x)}{a x+b-1} .
$$

Proposition 3 (Sum of first $n$ terms with odd indices). The sum of first $n$ terms with odd indices of generalized Fibonacci polynomials is given by

$$
\sum_{k=0}^{n-1} R_{2 k+1}(x)=\frac{R_{2 n+1}(x)-b^{2} R_{2 n-1}(x)+b\left(R_{1}(x)-a x R_{0}(x)\right)-R_{1}(x)}{a^{2} x^{2}-b^{2}+2 b-1} .
$$

Proof. Using Binet's formula (8), we have

$$
\begin{aligned}
\sum_{k=0}^{n-1} R_{2 k+1}(x) & =\sum_{k=0}^{n-1}\left(A \alpha^{2 k+1}(x)+B \beta^{2 k+1}(x)\right) \\
& =A \sum_{k=0}^{n-1} \alpha^{2 k+1}(x)+B \sum_{k=0}^{n-1} \beta^{2 k+1}(x) \\
& =\frac{A\left(\alpha^{2 n+1}(x)-\alpha(x)\right)}{\alpha^{2}(x)-1}+\frac{B\left(\beta^{2 n+1}(x)-\beta(x)\right)}{\beta^{2}(x)-1} .
\end{aligned}
$$

Thus

$$
\begin{aligned}
\sum_{k=0}^{n-1} R_{2 k+1}(x)= & \frac{A \alpha(x)+B \beta(x)-\alpha(x) \beta(x)(A \beta(x)+B \alpha(x))}{(\alpha(x) \beta(x))^{2}-\alpha^{2}(x)-\beta^{2}(x)+1} \\
& -\frac{A \alpha^{2 n+1}(x)+B \beta^{2 n+1}(x)+(\alpha(x) \beta(x))^{2}\left(A \alpha^{2 n-1}(x)+B \beta^{2 n-1}(x)\right)}{(\alpha(x) \beta(x))^{2}-\alpha^{2}(x)-\beta^{2}(x)+1} .
\end{aligned}
$$

Since $\alpha(x) \beta(x)=-b$ and $\alpha^{2}(x)+\beta^{2}(x)=a^{2} x^{2}+2 b$ then using (8), (11) and (12), we obtain

$$
\sum_{k=0}^{n-1} R_{2 k+1}(x)=\frac{R_{2 n+1}(x)-b^{2} R_{2 n-1}(x)+b\left(R_{1}(x)-a x R_{0}(x)\right)-R_{1}(x)}{a^{2} x^{2}-b^{2}+2 b-1} .
$$

Proposition 4 (Sum of first $n$ terms with even indices). The sum of first $n$ terms of generalized Fibonacci sequences with even indices is given by

$$
\sum_{k=0}^{n-1} R_{2 k}(x)=\frac{R_{2 n}(x)-b^{2} R_{2 n-2}(x)+\left(a^{2} x^{2} R_{0}(x)-a x R_{1}(x)+b R_{0}(x)\right)-R_{0}(x)}{a^{2} x^{2}-b^{2}+2 b-1} .
$$


Proof. Using Binet's formula (8), we have

$$
\sum_{k=0}^{n-1} R_{2 k}(x)=\sum_{k=0}^{n-1}\left(A \alpha^{2 k}(x)+B \beta^{2 k}(x)\right)=A \sum_{k=0}^{n-1} \alpha^{2 k}(x)+B \sum_{k=0}^{n-1} \beta^{2 k}(x)=\frac{A\left(\alpha^{2 n}(x)-1\right)}{\alpha^{2}(x)-1}+\frac{B\left(\beta^{2 n}(x)-1\right)}{\beta^{2}(x)-1} .
$$

Hence

$$
\sum_{k=0}^{n-1} R_{2 k}(x)=\frac{A+B-\left(A \beta^{2}(x)+B \alpha^{2}(x)\right)-\left(A \alpha^{2 n}(x)+B \beta^{2 n}(x)\right)}{(\alpha(x) \beta(x))^{2}-\alpha^{2}(x)-\beta^{2}(x)+1}+\frac{(\alpha(x) \beta(x))^{2}\left(A \alpha^{2 n-2}(x)+B \beta^{2 n-2}(x)\right)}{(\alpha(x) \beta(x))^{2}-\alpha^{2}(x)-\beta^{2}(x)+1} .
$$

Since $\alpha^{2}(x)+\beta^{2}(x)=a^{2} x^{2}+2 b$, and $\alpha(x) \beta(x)=-b$, then using (8), (10) and (13), we obtain

$$
\sum_{k=0}^{n-1} R_{2 k}(x)=\frac{R_{2 n}(x)-b^{2} R_{2 n-2}(x)+\left(a^{2} x^{2} R_{0}(x)-a x R_{1}(x)+b R_{0}(x)\right)-R_{0}(x)}{a^{2} x^{2}-b^{2}+2 b-1} .
$$

Proposition 5. For every positive integer $n$, we have

$$
\sum_{k=1}^{n} R_{3 k}(x)=\frac{R_{3 n+3}(x)+b^{3} R_{3 n}(x)-R_{3}(x)-b R_{0}(x)}{a^{3} x^{3}+b^{3}+3 a b x-1} .
$$

Proof. By Binet's formula (8), we have

$$
\sum_{k=1}^{n} R_{3 k}(x)=\sum_{k=1}^{n}\left(A \alpha^{3 k}(x)+B \beta^{3 k}(x)\right)=\frac{A \alpha^{3}(x)\left(\alpha^{3 n}(x)-1\right)}{\alpha^{3}(x)-1}+\frac{B \beta^{3}(x)\left(\beta^{3 n}(x)-1\right)}{\beta^{3}(x)-1} .
$$

Thus

$$
\begin{aligned}
\sum_{k=0}^{n} R_{3 k}(x)= & \frac{\left(A \alpha^{3}(x)+B \beta^{3}(x)\right)-\left(A \alpha^{3} \beta^{3}+B \beta^{3} \alpha^{3}\right)}{(\alpha(x) \beta(x))^{3}-\alpha^{3}(x)-\beta^{3}(x)+1} \\
& +\frac{\left(A \alpha^{3 n+3}(x) \beta^{3}(x)+B \beta^{3 n+3}(x) \alpha^{3}(x)\right)-\left(A \alpha^{3 n+3}(x)+B \beta^{3 n+3}(x)\right)}{(\alpha(x) \beta(x))^{3}-\alpha^{3}(x)-\beta^{3}(x)+1} . \\
= & \frac{\left(A \alpha^{3}(x)+B \beta^{3}(x)\right)-\alpha^{3}(x) \beta^{3}(x)(A+B)}{(\alpha(x) \beta(x))^{3}-\alpha^{3}(x)-\beta^{3}(x)+1} \\
& +\frac{\alpha^{3}(x) \beta^{3}(x)\left(A \alpha^{3 n}(x)+B \beta^{3 n}(x)\right)-\left(A \alpha^{3 n+3}(x)+B \beta^{3 n+3}(x)\right)}{(\alpha(x) \beta(x))^{3}-\alpha^{3}(x)-\beta^{3}(x)+1} .
\end{aligned}
$$

Since $\alpha^{3}(x)+\beta^{3}(x)=a^{3} x^{3}+3 a b x$, and $\alpha(x) \beta(x)=-b$, then by Equations (8) and (10), we get

$$
\sum_{k=1}^{n} R_{3 k}(x)=\frac{R_{3 n+3}(x)+b^{3} R_{3 n}(x)-R_{3}(x)-b R_{0}(x)}{a^{3} x^{3}+b^{3}+3 a b x-1} .
$$

Hence the proof.

Proposition 6. For every positive integer $n$, we have

$$
\sum_{k=1}^{n} R_{3 k-1}(x)=\frac{R_{3 n+2}(x)+b^{3} R_{3 n-1}(x)+b^{2}\left(a x R_{0}(x)-R_{1}(x)\right)-R_{2}(x)}{a^{3} x^{3}+b^{3}+3 a b x-1} .
$$

Proof. By Binet's formula (8), we have

$$
\sum_{k=1}^{n} R_{3 k-1}(x)=\sum_{k=1}^{n}\left(A \alpha^{3 k-1}(x)+B \beta^{3 k-1}(x)\right)=\frac{A \alpha^{2}(x)\left(\alpha^{3 n}(x)-1\right)}{\alpha^{3}(x)-1}+\frac{B \beta^{2}(x)\left(\beta^{3 n}(x)-1\right)}{\beta^{3}(x)-1} .
$$


This sum gives

$$
\begin{aligned}
\sum_{k=1}^{n} R_{3 k-1}(x)= & \frac{A \alpha^{2}(x)+B \beta^{2}(x)-\left(A \alpha^{2}(x) \beta^{3}(x)+B \alpha^{3}(x) \beta^{2}(x)\right)}{(\alpha(x) \beta(x))^{3}-\alpha^{3}(x)-\beta^{3}(x)+1} \\
& +\frac{A \alpha^{3 n+2}(x) \beta^{3}(x)+B \beta^{3 n+2}(x) \alpha^{3}(x)-A \alpha^{3 n+2}(x)+B \beta^{3 n+2}(x)}{(\alpha(x) \beta(x))^{3}-\alpha^{3}(x)-\beta^{3}(x)+1} \\
= & \frac{A \alpha^{2}(x)+B \beta^{2}(x)-(\alpha(x) \beta(x))^{2}(A \beta(x)+B \alpha(x))}{(\alpha(x) \beta(x))^{3}-\alpha^{3}(x)-\beta^{3}(x)+1} \\
& +\frac{(\alpha(x) \beta(x))^{3}\left(A \alpha^{3 n-1}(x)+B \beta^{3 n-1}(x)\right)-A \alpha^{3 n+2}(x)+B \beta^{3 n+2}(x)}{(\alpha(x) \beta(x))^{3}-\alpha^{3}(x)-\beta^{3}(x)+1} .
\end{aligned}
$$

Since $\alpha^{3}(x)+\beta^{3}(x)=a^{3} x^{3}+3 a b x$, and $\alpha(x) \beta(x)=-b$, then making use of (8) and (12), we obtain

$$
\sum_{k=1}^{n} R_{3 k-1}(x)=\frac{R_{3 n+2}(x)+b^{3} R_{3 n-1}(x)+b^{2}\left(a x R_{0}(x)-R_{1}(x)\right)-R_{2}(x)}{a^{3} x^{3}+b^{3}+3 a b x-1} .
$$

Proposition 7. For every positive integer $n$, we have

$$
\sum_{k=1}^{n} R_{3 k-2}(x)=\frac{R_{3 n+1}(x)+b^{3} R_{3 n-2}(x)-b\left(a^{2} x^{2} R_{0}(x)-a x R_{1}(x)+b R_{0}(x)\right)-R_{1}(x)}{a^{3} x^{3}+b^{3}+3 a b x-1} .
$$

Proof. By Binet's formula (8), we get

$$
\begin{aligned}
\sum_{k=1}^{n} R_{3 k-2}(x) & =\sum_{k=1}^{n}\left(A \alpha^{3 k-2}(x)+B \beta^{3 k-2}(x)\right) \\
& =A \sum_{k=1}^{n} \alpha^{3 k-2}(x)+B \sum_{k=1}^{n} \beta^{3 k-2}(x) \\
& =\frac{A \alpha(x)\left(\alpha^{3 n}(x)-1\right)}{\alpha^{3}(x)-1}+\frac{B \beta(x)\left(\beta^{3 n}(x)-1\right)}{\beta^{3}(x)-1} .
\end{aligned}
$$

This Sum gives

$$
\begin{aligned}
\sum_{k=1}^{n} R_{3 k-2}(x)= & \frac{A \alpha(x)+B \beta(x)-A \alpha \beta^{3}(x)+B \beta \alpha^{3}(x)}{(\alpha(x) \beta(x))^{3}-\alpha^{3}(x)-\beta^{3}(x)+1} \\
& +\frac{A \alpha^{3 n+1}(x) \beta^{3}(x)+B \beta^{3 n+1}(x) \alpha^{3}(x)-A \alpha^{3 n+1}(x)+B \beta^{3 n+1}(x)}{(\alpha(x) \beta(x))^{3}-\alpha^{3}(x)-\beta^{3}(x)+1} \\
= & \frac{A \alpha(x)+B \beta(x)-\alpha(x) \beta(x)\left(A \alpha \beta^{2}(x)+B \alpha^{2}(x)\right)}{(\alpha(x) \beta(x))^{3}-\alpha^{3}(x)-\beta^{3}(x)+1} \\
& +\frac{(\alpha(x) \beta(x))^{3}\left(A \alpha^{3 n-2}(x)+B \beta^{3 n-2}(x)\right)-A \alpha^{3 n+1}(x)+B \beta^{3 n+1}(x)}{(\alpha(x) \beta(x))^{3}-\alpha^{3}(x)-\beta^{3}(x)+1} .
\end{aligned}
$$

Since $\alpha^{3}(x)+\beta^{3}(x)=a^{3} x^{3}+3 a b x$, and $\alpha(x) \beta(x)=-b$, then making use of (8) and (13), we obtain

$$
\sum_{k=1}^{n} R_{3 k-2}(x)=\frac{R_{3 n+1}(x)+b^{3} R_{3 n-2}(x)-b\left(a^{2} x^{2} R_{0}(x)-a x R_{1}(x)+b R_{0}(x)\right)-R_{1}(x)}{a^{3} x^{3}+b^{3}+3 a b x-1} .
$$

Theorem 3 (Generalized identity). Let $R_{n}(x)$ be the $n^{\text {th }}$ generalized Fibonacci polynomials. Then 


$$
\begin{aligned}
& R_{m}(x) R_{n}(x)-R_{m-k}(x) R_{n+k}(x) \\
& =(-b)^{m-k}\left[\frac{\left(R_{1}(x) R_{k}(x)-R_{0}(x) R_{k+1}(x)\right)\left(R_{1}(x) R_{n-m+k}(x)-R_{0}(x) R_{n-m+k+1}(x)\right.}{\left.R_{1}^{2}(x)-R_{0}(x) R_{2}(x)\right)}\right],
\end{aligned}
$$

where $n>m \geq k \geq 1$.

Proof. Using Binet's formula (8) to the left hand side, we have

$$
\begin{aligned}
& \text { LHS }=\left(A \alpha^{m}(x)+B \beta^{m}(x)\right)\left(A \alpha^{n}(x)+B \beta^{n}(x)\right)-\left(A \alpha^{m-k}(x)+B \beta^{m-k}(x)\right)\left(A \alpha^{n+k}(x)+B \beta^{n+k}(x)\right) \\
& =A B\left(\alpha^{k}(x)-\beta^{k}(x)\right)\left[\frac{\alpha^{m}(x) \beta^{n}(x)}{\alpha^{k}(x)}-\frac{\alpha^{n}(x) \beta^{m}(x)}{\beta^{k}(x)}\right] \\
& =A B \frac{\left(\alpha^{k}(x)-\beta^{k}(x)\right)}{(\alpha(x) \beta(x))^{k}}\left(\alpha^{m}(x) \beta^{n+k}(x)-\alpha^{n+k}(x) \beta^{m}(x)\right) \\
& =-A B(-b)^{m-k}\left(\alpha^{k}(x)-\beta^{k}(x)\right)\left(\alpha^{n-m+k}(x)-\beta^{n-m+k}(x)\right) \text {. } \\
& R_{m}(x) R_{n}(x)-R_{m-k}(x) R_{n+k}(x)=\frac{R_{1}^{2}(x)-R_{0}(x) R_{2}(x)}{(\alpha(x)-\beta(x))^{2}}(-b)^{m-k}\left[\left(\alpha^{k}(x)-\beta^{k}(x)\right)\left(\alpha^{n-m+k}(x)-\beta^{n-m+k}(x)\right)\right] \\
& =\left(R_{1}^{2}(x)-R_{0}(x) R_{2}(x)\right)(-b)^{m-k}\left[\left(\frac{\alpha^{k}(x)-\beta^{k}(x)}{\alpha(x)-\beta(x)}\right)\left(\frac{\alpha^{n-m+k}(x)-\beta^{n-m+k}(x)}{\alpha(x)-\beta(x)}\right)\right] .
\end{aligned}
$$

From

$$
\frac{\alpha^{k}(x)-\beta^{k}(x)}{\alpha(x)-\beta(x)}=\frac{R_{1}(x) R_{k}(x)-R_{0}(x) R_{k+1}(x)}{R_{1}^{2}(x)-R_{0}(x) R_{2}(x)}
$$

and

$$
\frac{\alpha^{n-m+k}(x)-\beta^{n-m+k}(x)}{\alpha(x)-\beta(x)}=\frac{R_{1}(x) R_{n-m+k}(x)-R_{0}(x) R_{n-m+k+1}(x)}{R_{1}^{2}(x)-R_{0}(x) R_{2}(x)},
$$

we obtain our desired result.

Corollary 1 (Catalan's identity). If $m=n$ in the generalized identity (17), we obtain

$$
R_{n}^{2}(x)-R_{n-k}(x) R_{n+k}(x)=\frac{(-b)^{m-k}}{R_{1}^{2}(x)-R_{0}(x) R_{2}(x)}\left[R_{1}(x) R_{k}(x)-R_{0}(x) R_{k+1}(x)\right]^{2},
$$

where $n>k \geq 1$.

Corollary 2 (Cassini's identity). If $m=n$ and $k=1$ in the generalized identity (17), we obtain

$$
R_{1}^{2}(x)-R_{n-1}(x) R_{n+1}(x)=(-b)^{n-1}\left[R_{1}^{2}(x)-R_{0}(x) R_{2}(x)\right],
$$

for $n \geq 1$.

Corollary 3 (d'Ocagne's identity). If $n=m, m=n+1$ and $k=1$ in the generalized identity (17), we obtain

$$
R_{m}(x) R_{n+1}(x)-R_{m+1}(x) R_{n}(x)=(-b)^{n}\left[R_{1}(x) R_{m-n}(x)-R_{0}(x) R_{m-n+1}(x)\right],
$$

where $m>n \geq 1$. 


\section{Conclusion}

In this paper generalized Fibonacci polynomials is defined by recurrence relations (6). Binet's formula (8) and generating function of these polynomials (15) are derived. Further explicit sum formula, sum of first $n$ terms, sum of first $n$ terms with (odd or even )indices and generalized identity (17) from which we obtain Catalan's identity, Cassini's identity and d'Ocagne's identity are also derived.

Conflicts of Interest: "The author declares no conflict of interest."

\section{References}

[1] Koshy, T. (2011). Fibonacci and Lucas numbers with applications. Wisley-Interscience Publications, New York.

[2] Horadam, A. F., \& Mahon, B. J. M.(1985). Pell and Pell Lucas Polynomials. The Fibonacci Quartely, 23(1), 7-20.

[3] Horzum, T. (2009). On some properties of Horadam polynomials. International Mathematical Forum, 4(25), $1243-1252$.

[4] Rathore, G. P. S. , Sikhwal, O , \& Choundry, R. (2016). Generalized Fibonacci polynomials and some identities. International Journal of Computer Applications, 153(12), 4-8.

[5] Singh, M. Gupta Y. K., \& Sikhwal, O. (2014). Generalized Fibonacci-like polynomials and some identities. Global Journal of Mathematical Anlysis, 2(4), 249-258.

[6] Singh, B., Bhatnagar, S., \& Sikhwal, O. (2013). Fibonacci-like Polynomials and Some identities. International Journal of Advanced Mathematical Sciences, 1(3), 152-157.

[7] Singh, M., Sikhwal, O., \& Gupta, Y. K. (2014). Generalized Fibonacci-Lucas Polynomials. International journal of Advanced Mathematical Sciences, 2(1), 81-87.

[8] Sloane, N. J. A. The On-Line Encyclopedia of Integer Sequences (OEIS). Available online at http:/ / oeis.org.

(C) 2020 by the authors; licensee PSRP, Lahore, Pakistan. This article is an open access article distributed under the terms and conditions of the Creative Commons Attribution (CC-BY) license (http://creativecommons.org/licenses/by/4.0/). 